\title{
Características psicológicas relacionadas con el rendimiento deportivo de runners adultos pertenecientes a clubes de Concepción
}

\author{
Psychological characteristics related to the athletic performance of adult runners \\ affiliated to clubs of Concepcion
}

\author{
*Cristián Luarte Rocha, **María Elena Seguel López, \\ ***Luis Felipe Castelli Correia de Campos
}

Luarte, C., Seguel, M., Castelli, L. (2018). Características psicológicas relacionadas con el rendimiento deportivo de runners adultos pertenecientes a clubes de Concepción. Revista Ciencias de la Actividad Física UCM, No 19(2) julio-diciembre, 1-8. DOI: http://doi.org/10.29035/rcaf.19.2.7

\section{RESUMEN}

El presente estudio consistió en identificar las características psicológicas del rendimiento deportivo en atletas Runners de clubes pertenecientes a la ciudad de Concepción, Chile. El estudio fue de diseño no experimental-transversal de alcance descriptivo. Para recolectar los datos se aplicó el cuestionario de características psicológicas relacionadas con el rendimiento deportivo (CPRD). La información que entregó el cuestionario CPRD, señala que los resultados fluctúan sobre los promedios esperados y que son considerados normales según el Baremo estándar: "CPRD" control estrés 80,00\%, influencia de evaluación $85,00 \%$, motivación $70,00 \%$, autoconfianza $73,08 \%$, influencia del entrenador $76,80 \%$, habilidad para establecer objetivos $72,12 \%$, cohesión social $86,30 \%$ y ansiedad previa competencia $48,32 \%$. De acuerdo a los resultados, su rendimiento deportivo es satisfactorio. Dentro de las características psicológicas mencionadas, se pueden destacar; la ansiedad, atención, autoconfianza, motivación, estrés y cohesión social. Todas ellas son características que afectan significativamente el rendimiento deportivo.

\section{PALABRAS CLAVE}

Runners amateur, características psicológicas, rendimiento deportivo.

\begin{abstract}
The following study consisted of identifying the psychological characteristics of the amateur runners belonging to the clubs of Concepcion Chile. The research was quantitative of non-experimental transversal of descriptive scope. To collect data a questionnaire, involving the psychological characteristics associated with sport performance (CPRD) was applied. The information obtained by the CPRD show that the results are in average of the expected results, and are considered normal according to standar scale: "CPRD" stress control $80,00 \%$, influence of evaluation $85,00 \%$, motivation $70,00 \%$, selfconfidence $73,08 \%$, coach influence $76,80 \%$, set goals $72,12 \%$, social cohesion $86,30 \%$, anxiety before competition $48,32 \%$. According to these results, their sports performance is satisfactory. Alongside the psychological characteristics previously mentioned it could be highlighted anxiety, attention, selfconfidence, motivation, stress and social cohesion. These characteristics affect significantly sports performance.
\end{abstract}

\section{Key words}

Amateur runners, psychological characteristics, sports performance. 


\section{INTRODUCCIÓN}

El running es una modalidad deportiva, considerada como un fenómeno social, ya que, ha tenido un progresivo crecimiento en los últimos años, mayoritariamente por personas que lo practican de forma amateurs. Esta investigación responde a una dimensión importante en el rendimiento de un atleta-deportista, como lo son; las características psicológicas, tales como; motivación, estrés, ansiedad y autoconfianza. Según Cox (2009) la psicología del deporte es el estudio de los factores psicológicos y emocionales sobre el rendimiento deportivo y el efecto de las actividades sobre estos factores, es una ciencia que entrega conocimiento especializado del comportamiento humano con una metodología para evaluar, comprender y modificar aspectos relacionados con el campo de estudio. A su vez, Buceta (1998) señala que, el funcionamiento psicológico del deportista, puede influir de forma positiva o negativa al rendimiento, es por ello que, debe ser integrada dentro del entrenamiento y la formación global del deportista.

En función a lo anterior, entendemos como un componente importante del rendimiento deportivo, son las características psicológicas que posee un atleta, ya que, según éstas, el rendimiento puede ser positivo o negativo. Entendemos el rendimiento positivo cuando el deportista muestra un buen resultado en una competencia o en un control dentro de su planificación. Por su parte, el rendimiento será negativo cuando el individuo no es capaz de cumplir con lo esperado al momento de enfrentar una competición, es decir, no siempre los resultados son productos de los componentes biocinéticos; tales como: velocidad, fuerza, resistencia. Por el contrario, en muchas ocasiones, es la dimensión psicológica la que incide en el resultado, y de acuerdo a los contextos, ésta puede afectar; negativamente al no conseguir lo que se espera o por el contrario lograr lo que se había planeado e incluso superarlo (Bandura, 1997).

Dentro de las variables psicológicas que se consideraron importantes para este estudio están: la ansiedad, que es el mecanismo adaptativo que ayuda en la activación de conductas de defensa y ayuda a superar dificultades. La atención, que es la capacidad cognitiva que permite elegir el tipo de información deseada, según el momento que aprende, se perfecciona o se desarrolla. La autoconfianza que, según Bandura (1977) es el estado interno de fortaleza psicológica que implica un conocimiento real de las dificultades del objetivo y de los recursos que poseen. El estrés que, prepara al organismo y provoca cambios fisiológicos. Weineck (2000) señala que, el estrés ha estado con el ser humano desde principios de su existencia de forma similar a lo largo de los años, donde participan principalmente las hormonas adrenalina y noradrenalina, las que provocan cambios fisiológicos que preparan al individuo para responder de mejor manera a estímulos que son considerados peligrosos o donde se requiere una mayor respuesta a situaciones del ambiente. Esto tiene una fuerte influencia en el rendimiento deportivo de un atleta. La motivación que, es culpable de la pertinencia y del esfuerzo. Lo anterior se vincula estrechamente a las emociones que, condicionan las vivencias. Estas son reacciones psicofisiológicas a las relaciones interpersonales y tienen poca duración (miedo, ira, sorpresa) en cambio los sentimientos son más profundos, duraderos, menos intensos y se dan a nivel psicológico (amor, rencor, cariño), percepción de competencia si poseen una alta percepción, mayor participación, persistencia, compromiso y elección, tolerancia al bajo rendimiento siendo éste último el responsable de la deserción, cuando el temor al fracaso es, un fuerte, causa que eviten la actividad (Cox, 2009).

El objetivo del presente estudio fue identificar y describir las características psicológicas relacionadas con el rendimiento deportivo, en runners adultos de clubes amateurs de la ciudad de Concepción.

\section{METODOLOGÍA}

La investigación tiene un enfoque cuantitativo, recolectando los datos, a través de instrumentos estandarizados. El diseño es de tipo no experimental-transversal de alcance descripti- 
vo, es decir, no hay manipulación de variables, se observan en un momento y se describen características de la muestra. La muestra es intencionada no probabilística: la elección no depende de la probabilidad, sino de las características de la investigación. Muestra de 52 adultos 27 mujeres y 25 hombres entre 18 y 70 años. La técnica de análisis estadístico es de tipo descriptivo, con representación mediante gráficos. Se utilizó el programa CPRD, para recolectar y para analizar los datos que se obtuvieron, a través del cuestionario del mismo programa. La creación del instrumento tomó como punto de partida el Psychological Skills Inventory for Sport (PSIS). Con la posterior traducción y modificación al español, surge el Cuestionario de Características Psicológicas relacionadas con el Rendimiento Deportivo (CPRD) (Gimeno \& Buceta, 2010). El instrumento se compone de 55 ítems, comprimido en 5 factores que presentan, en la mayoría de los casos, coeficiente de consistencia interna adecuada: control del estrés (alfa $=0,88$ ), cohesión del equipo (alfa $=0,78$ ) evaluación de desempeño (alfa $=0,72)$, motivación (alfa $=$ $0,67)$ y capacidad mental (alfa $=0,34)$. Cuando se analizó el CPRD como un todo, se observó un coeficiente de consistencia interna de 0,85 . La escala de medida utilizada es del tipo likert, con valores que varían de 1 (totalmente en desacuerdo) a 5 (totalmente de acuerdo). Se destaca que, si algún ítem no fue comprendido por los corredores, incluso después de la explicación previa, los sujetos podrían elegir la opción NE (no entiendo). La estructura factorial del cuestionario CPRD está definida por cinco escalas, en lugar de las seis contenidas en el cuestionario original PSIS. Estas escalas aglutinan un total de 55 ítems e incluyen variables psicológicas que están asociadas al rendimiento deportivo. Las variables fueron: control del estrés, influencia de la evaluación del rendimiento, motivación, habilidad mental, y cohesión de equipo; y otras en base a su relación con éstas: la autoconfianza en relación con el control del estrés; la influencia de la evaluación y la motivación, el nivel de activación y la atención en relación con el control del estrés y la influencia de la evaluación. El cuestionario se efectuó, en un lugar cómodo, en el cual los atletas recibieron la información, se aclaran dudas y se otorga el tiempo necesario para sus respuestas.

\section{RESULTADOS Y DISCUSIÓN}

Los valores obtenidos de las características psicológicas globales se presentan en la Tabla 1 .

\section{Tabla 1}

Características psicológicas globales de runners adultos.

\begin{tabular}{lccc}
\hline & General $(\mathbf{m} \pm$ de) & Masculino $(\mathbf{m} \pm \mathbf{d e})$ & Femenino $(\mathbf{m} \pm$ de) \\
\hline Control del Estrés (CE) & $56,4 \pm 1,9$ & $57,9 \pm 2,6$ & $55,0 \pm 2,7$ \\
\hline Evaluación del Rendimiento (ER) & $32,8 \pm 1,2$ & $33,6 \pm 1.9$ & $31,9 \pm 1,6$ \\
\hline Motivación (MO) & $21,9 \pm 0,8$ & $22,0 \pm 1,2$ & $21,6 \pm 1,1$ \\
\hline Habilidad Mental (HM) & $23,7 \pm 0,7$ & $23,2 \pm 1,1$ & $24,2 \pm 0,8$ \\
\hline Cohesión en Equipo (COE) & $18,3 \pm 0,6$ & $18,2 \pm 0,9$ & $18,3 \pm 0,9$ \\
\hline
\end{tabular}

Los datos obtenidos de 52 sujetos que participaron en el estudio se observa que, el control de estrés es el valor que se encuentra con una puntuación centil más elevada $(\mathrm{PC}=56,4) \sin$ grandes diferencias entre sexos, siendo levemente superior en varones. La variable o baremo con menor puntuación es la cohesión de equipo $(\mathrm{PC}=18,3)$. A diferencia de los resulta- dos que se entregan en el estudio de Ureña-Bonilla (en Gimeno \& Buceta, 2010) realizado a jugadores profesionales de fútbol y baloncesto que, indican que estos deportistas tienen baja tolerancia al estrés en la competencia deportiva. Los atletas-deportistas evaluados en este estudio, muestran que el control de estrés (CE) está en parámetros aceptables, superior a la 
media según el baremo estandar que entrega el programa. $(\mathrm{PC}=50.00)$.

Resulta interesante el análisis de los resultados de la variable cohesión de equipo (COE) debido a que, el Running es un deporte de desempeño individual, pero que se desarrolla en contextos de participación grupal, por lo cual, la cohesión centrada en el objetivo del grupo como en deportes grupales no posee mayor relevancia, pero que, al entrenar en grupo, es de gran importancia. Los resultados de esta variable $(\mathrm{PC}=18.3)$, se encuentran levemente superior en comparación a Mahamud, Tuero \& Márquez. (2005) estudio realizado entre atletas de diferentes disciplinas de participación individual, con ( $\mathrm{PC}=17.5)$. De la misma forma, las puntuaciones levemente inferiores según el estudio de Sánchez, Carmona \& Lopa (en Gimeno \& Buceta, 2010), que presentó valores de $(P C=20)$ en atletas de modalidades individuales.

Estudios efectuados por Bray y Whaley (en Gimeno \& Buceta, 2010) corroboran que la co- hesión social impulsa a los individuos a esforzarse más, que posee una gran relación con el rendimiento individual objetivo, además que el esfuerzo realizado sirve como mediador en la relación con otros individuos, es decir, que la cohesión social no tiene un efecto directo en el rendimiento, pero sí tiene un efecto indirecto a través del esfuerzo. De igual forma, North y Cols (en Gimeno \& Buceta, 2010), manifiestan que la interacción social cuando está ligada al ejercicio con amigos y colegas es placentera y ayuda a mejorar la salud mental, por ende, mejora el rendimiento.

En la Tabla 2 se presentan los valores de puntuación centil (PC) en cada componente de las variables psicológicas globales; CE - Control del Estrés; ER - Evaluación del Rendimiento; MO - Motivación; COE - Cohesión de Equipo. Los principales resultados identificaron valores más altos de (PC) para los factores de CE-autoconfianza (73.1), ER-conducta del entrenador (76.8), MO- habilidad establecimiento de objetivos (72.1) y COE- Cohesión Social (86.3).

\section{Tabla 2}

Caracterización psicológica de la muestra para las variables de control de estrés, evaluación del rendimiento, motivación y cohesión de equipo.

\begin{tabular}{|c|c|c|c|c|}
\hline \multicolumn{2}{|c|}{ Variables Psicológicas } & \multirow{2}{*}{$\begin{array}{c}\begin{array}{c}\text { General } \\
(\mathrm{m} \pm \mathrm{de})\end{array} \\
64,4 \pm 0,6\end{array}$} & \multirow{2}{*}{$\begin{array}{c}\begin{array}{c}\text { Masculino } \\
(\mathrm{m} \pm \mathrm{de})\end{array} \\
68,4 \pm 0,8\end{array}$} & \multirow{2}{*}{$\begin{array}{c}\begin{array}{c}\text { Femenino } \\
(\mathrm{m} \pm \mathrm{de})\end{array} \\
60,7 \pm 0,8\end{array}$} \\
\hline & Control Ansiedad & & & \\
\hline CE & Autoconfianza & $73,1 \pm 1,0$ & $74,9 \pm 1,2$ & $71,4 \pm 1,5$ \\
\hline & Atención & $71,6 \pm 0,6$ & $71,6 \pm 0,9$ & $71,7 \pm 0,8$ \\
\hline \multirow{5}{*}{ ER } & Control Ansiedad & $56,1 \pm 0,4$ & $60,3 \pm 0,6$ & $52,2 \pm 0,5$ \\
\hline & Autoconfianza & $62,5 \pm 0,2$ & $58,0 \pm 0,3$ & $66,7 \pm 0,3$ \\
\hline & Motivación & $72,1 \pm 0,2$ & $64,0 \pm 0,2$ & $79,6 \pm 0,2$ \\
\hline & Atención & $69,7 \pm 0,4$ & $76,0 \pm 0,5$ & $63,9 \pm 0,6$ \\
\hline & Conducta del Entrenador & $76,8 \pm 0,4$ & $77,5 \pm 0,6$ & $76,2 \pm 0,5$ \\
\hline \multirow{3}{*}{$\mathrm{MO}$} & Motivación Básica & $69,6 \pm 0,5$ & $68,8 \pm 0,7$ & $70,4 \pm 0,6$ \\
\hline & Motivación Cotidiana & $66,0 \pm 0,4$ & $65,3 \pm 0,5$ & $66,7 \pm 0,5$ \\
\hline & Habilidad Establecimiento de Objetivos & $72,1 \pm 0,1$ & $75,0 \pm 0,2$ & $69,4 \pm 0,2$ \\
\hline \multirow{3}{*}{$\mathrm{COE}$} & Espíritu de Equipo & $86,1 \pm 0,3$ & $82,0 \pm 0,4$ & $89,8 \pm 0,3$ \\
\hline & Individual vs Colectivo & $55,8 \pm 0,3$ & $57,0 \pm 0,4$ & $54,6 \pm 0,4$ \\
\hline & Cohesión Social & $86,3 \pm 0,3$ & $88,0 \pm 0,3$ & $84,7 \pm 0,3$ \\
\hline
\end{tabular}

Nota: CE - Control del estrés; ER - Evaluación del rendimiento; MO - Motivación; COE - Cohesión de equipo. 
$\mathrm{Al}$ analizar la variable, CE-control de estrés, se observa que el control de ansiedad se encuentra con una $(\mathrm{PC}=64,4)$ considerada baja en relación a la atención y a la autoconfianza, siendo esta última la que posee mejor puntuación. Sin embargo, esta puntuación contrasta a estudios de Gimeno, \& Buceta (2010) con atletas españoles de mejor nivel competitivo y de mayor experiencia. Se entiende que los atletas del estudio, no están sometido a una carga psicológica competitiva. Producto de ello, y al poseer una alta autoconfianza, nos demuestran que confían en los recursos con los que cuentan para afrontar situaciones estresantes. También denotan una gran habilidad de mantener su concentración en situaciones del mismo tipo (atención) y a pesar de que posee menor puntuación, también tienen un buen control de ansiedad, no obstante, la medición indica que necesitarán mayor trabajo.

A su vez, en la variable ER-Evaluación del rendimiento, la influencia o conducta del entrenador tiene una mayor puntuación $(\mathrm{PC}=76,8)$ frente a la situación de competencia, indicando que, para el grupo de deportistas es fundamental lo que dice, hace o demuestra, situación que llama la atención si la analizamos con otras variables que predisponen al deportista. Se podría esperar que la variable motivación $(\mathrm{PC}=72,1)$ fuera la más alta puntuación en este baremo. También se observa que la motivación dentro de este grupo afecta de sobremanera el rendimiento frente a una evaluación y como se demuestra en tablas anteriores, la más baja puntuación es el control de ansiedad que, se entiende como la capacidad del atleta en controlar momentos de ansiedad y reacciones no beneficiosas para el rendi- miento. Autores como Anderson (en Gimeno \& Buceta, 2010) define a la ansiedad como; un patrón de conducta complejo, que posee sentimientos subjetivos de tensión, acompañado de actividad fisiológica. En la variable MO-Motivación, se infiere que, el grupo observado posee una alta habilidad para establecer objetivos $(\mathrm{PC}=72,1)$ seguida por la motivación básica y posteriormente la motivación cotidiana. Dentro de este grupo la habilidad para establecer objetivos es bastante elevada en comparación con estudios similares en deportistas mexicanos, Gimeno \& Buceta (2010), por lo cual se infiere que los sujetos en estudio, tienen facilidad para fijar las metas que desean alcanzar, sean estas a mediano o a largo plazo. La motivación básica $(\mathrm{PC}=69,6)$ es la segunda más alta, demostrando que este grupo de atletas poseen mucho interés en la actividad que realizan. La más baja, pero aun así sobre el promedio, es la motivación cotidiana $(\mathrm{PC}=66,0)$ que se refiere a la gratificación y el interés que entrega cada entrenamiento, competición o actividad a este grupo de atletas. Finalmente, y de acuerdo a los resultados obtenidos en la variable COE-Cohesión de equipo, se aprecia que, la cohesión social es la variable más alta $(\mathrm{PC}=86,3)$ levemente superior a la variable espíritu de equipo.

De acuerdo a los resultados expresados en la tabla 3 , se indican las variables que componen el cuestionario CPRD, estas son; CA - Control de Ansiedad; AC - Autoconfianza; AT - Atención y TBR - Tolerancia al bajo Rendimiento. A continuación, se presentan las puntuaciones obtenidas por estas variables que, tributan a las variables globales mencionadas en la Tabla 1 . 
Tabla 3

Caracterización Psicológica de la muestra para las variables de Control de Ansiedad, Autoconfianza, Atención y Tolerancia al bajo Rendimiento.

\begin{tabular}{llccc}
\hline \multirow{2}{*}{ Variables Psicológicas } & $\begin{array}{c}\text { General } \\
(\mathbf{m} \pm \mathbf{d e})\end{array}$ & $\begin{array}{c}\text { Masculino } \\
(\mathbf{m} \pm \mathbf{d e})\end{array}$ & $\begin{array}{c}\text { Femenino } \\
(\mathbf{m} \pm \mathbf{d e})\end{array}$ \\
\hline \multirow{2}{*}{ CA } & Previa a la Competición & $48,3 \pm 0,4$ & $54,2 \pm 0,5$ & $42,8 \pm 0,6$ \\
\cline { 2 - 5 } & Durante la Competición & $56,1 \pm 0,4$ & $60,3 \pm 0,6$ & $52,1 \pm 0,5$ \\
\cline { 2 - 5 } & Control de Ansiedad y Rendimiento & $60,1 \pm 0,2$ & $60,0 \pm 0,3$ & $61,1 \pm 0,3$ \\
\cline { 2 - 5 } & Habilidad para Controlar la Tensión & $68,3 \pm 0,2$ & $65,0 \pm 0,2$ & $71,3 \pm 0,2$ \\
\hline \multirow{2}{*}{ AC } & Aspectos Generales & $72,8 \pm 0,5$ & $76,8 \pm 0,5$ & $69,2 \pm 0,7$ \\
\cline { 2 - 5 } & Competición & $63,9 \pm 0,6$ & $63,4 \pm 0,9$ & $64,29 \pm 0,8$ \\
\cline { 2 - 5 } & Entrenamiento y Lesiones & $64,4 \pm 0,2$ & $62,0 \pm 0,2$ & $66,6 \pm 0,2$ \\
\hline \multirow{2}{*}{ AT } & Habilidad para estar Concentrado & $74,1 \pm 0,3$ & $76,0 \pm 0,4$ & $72,2 \pm 0,4$ \\
\cline { 2 - 5 } & Falta de Habilidad general & $72,6 \pm 0,3$ & $72,0 \pm 0,4$ & $73,1 \pm 0,4$ \\
\cline { 2 - 5 } & Motivos específicos para perdida de concentración & $70,2 \pm 0,7$ & $73,8 \pm 0,8$ & $66,8 \pm 1,0$ \\
\hline \multirow{2}{*}{ TBR } & Malas Actuaciones & $67,8 \pm 0,4$ & $70,7 \pm 0,6$ & $65,0 \pm 0,5$ \\
\cline { 2 - 5 } & Errores & $65,6 \pm 0,3$ & $68,0 \pm 0,4$ & $63,4 \pm 0,4$ \\
\cline { 2 - 5 } & Críticas & $86,5 \pm 0,1$ & $85,0 \pm 0,2$ & $87,9 \pm 0,1$ \\
\hline
\end{tabular}

Nota: CA - Control de ansiedad; AC - Autoconfianza; AT - Atención; TBR - Tolerancia al bajo rendimiento.

Respecto a la variable, CA-Control de ansiedad, la variable habilidad para controlar la tensión es la que obtiene la puntuación centil más elevada $(\mathrm{PC}=68,3)$ esto, reafirma estudios efectuados por Balager ; Weinberg y Gould, Buceta (en Gimeno, Buceta \& Pérez-Llantada, 2007) quienes, en estudios similares, junto con señalar que están dentro de las habilidades psicológicas importantes, muestran resultados cercanos con deportistas españoles, en modalidades individuales. Con una puntuación levemente inferior, encontramos la variable; control de ansiedad y rendimiento durante la competición. El más bajo es el control de ansiedad previa a la competencia $(\mathrm{PC}=56,1)$. Según estudios de Gimeno, \& Buceta, (2010), la ansiedad es la principal manifestación de estrés en el deporte de competición, por lo cual se espera una tendencia en gran parte de los atletas-deportistas, independiente del nivel de competencia. En este estudio, se observa que los atletas son capaces de controlar de buena manera la tensión, pero no logran controlar la ansiedad previa a la competencia, es decir, requieren implementar posibles necesidades de mejoramiento, por medio de estrategias y técnicas de autocontrol.
Si bien es sabido, la variable AC-Autoconfianza es trascendental en la evaluación psicológica de los deportistas de competición, para el estudio se consideró necesario su análisis, observando que, en los aspectos generales tiene una puntuación más elevada $(\mathrm{PC}=72,8)$. Esto último, demuestra que la autoconfianza general que, es la confianza que estos deportistas poseen en sus propios recursos para afrontar situaciones estresantes, es la más elevada, Buceta (1998). A su vez, podemos observar que, poseen una gran autoconfianza durante los entrenamientos o en situación de lesiones, es decir, saben que son circunstancias que pueden suceder y que deben ser atendidas de buena manera. Mientras que la que obtuvo más baja puntuación fue la autoconfianza durante la competencia $(\mathrm{PC}=63,9)$, es decir, que en estas situaciones es donde los atletas dudan más de sus habilidades para afrontar situaciones que podrían generar estrés.

El análisis de la variable AT-Atención, la habilidad de estar concentrado es la que obtiene más alta puntuación con $(\mathrm{PC}=74,1)$, seguido de la falta de habilidad general y los motivos específicos para la pérdida de concentración 
que, es el más bajo. Según estudios de Gimeno \& Buceta (2010) efectuados con deportistas mexicanos y españoles, es necesario abordar la concentración en todas sus variables, ya que nos ayudan a considerar déficits atencionales que podrían subsanarse con el entrenamiento y de esta forma evitar la pérdida de concentración ante esos estímulos concretos.

Las puntuaciones de los ítems que conforman la atención, nos indican que, se encuentran sobre el promedio, pero deben mejorar si desean ser comparados con deportistas que buscan mayores rendimientos. La habilidad para estar concentrados durante la competencia y durante los entrenamientos es la mejor evaluada ( $\mathrm{PC}=74,1)$ aunque se entiende que les afecta bastante, los motivos o situaciones específicas que les hace perder la concentración, ejemplo; cuando cometen un error, problemas familiares, de salud, emocionales y laborales. Respecto a lo mismo, estudios de Balaguer \& Castillo (1994), señalan que, el deportista o atleta debe ser capaz de seleccionar aquellos estímulos que son considerados relevantes para cada situación y no atender lo irrelevante o lo que es perjudicial.

Todos los deportes requieren de una combinación de procesamiento automático y de procesamiento controlado, puesto que a lo largo de la situación deportiva los atletas deben ejecutar muchas habilidades de forma automática; mientras que existen otras situaciones que precisan de la toma de decisiones y a la vez, procesar nueva información. Por tanto, necesitan de un procesamiento controlado. En este caso, como el running es un deporte de habilidades abiertas; es decir, donde el entorno cambia constantemente se necesita la combinación de ambos tipos de procesamientos, (autónomo y controlado) es por ello que, la atención es fundamental para todo deportista.

En la variable TBR-Tolerancia al bajo rendimiento, la variable crítica, es la de mayor puntuación centil, alcanzando un $(\mathrm{PC}=86,5)$ seguido por las malas actuaciones $(\mathrm{PC}=67,8)$ y finalmente los errores con una puntuación de $(\mathrm{PC}=65,6)$. Es muy importante detectar el nivel de estas variables, ya que, en estudios efectuado por Gimeno \& Buceta (2010), se infiere que la variable; tolerancia baja, podría estar relacionada con un exceso de perfeccionismo que, en el contexto de la competición, estaría influyendo negativamente en el funcionamiento del atleta-deportista. De la misma manera, una baja tolerancia al bajo rendimiento, podría relacionarse con una autoconfianza y una autoestima débil.

\section{CONCLUSIÓN}

La información que entregó el cuestionario CPRD: respecto a las características psicológicas de los deportistas runners adultos encuestados, de la ciudad de Concepción, indica que, los resultados fluctúan sobre los promedios esperados, considerado normal según el baremo estándar del programa, esto significa que, la muestra presenta las características que se esperaban. Dentro de las características psicológicas mencionadas, se destacan; la ansiedad, atención, autoconfianza, el estrés y la cohesión social, todas ellas, variables que afectan el rendimiento deportivo. De igual forma, el estudio arrojó que, tanto la ansiedad como el estrés en niveles normales son considerados positivos, pero cuando superan lo que el individuo es capaz de controlar, provoca errores técnicos en la ejecución de la actividad, impidiendo que el deportista consiga sus objetivos o metas propuestas. Por su parte, la variable autoconfianza, es una característica psicológica que, en exceso puede producir una sobrevaloración de sí mismo al momento de rendir, sin embargo, una baja valoración de sí mismo, impide desarrollar adecuadamente la práctica deportiva. Por otra parte, la atención del deportista y la cohesión social afectan de manera positiva al rendimiento deportivo. Por tanto, es fundamental que un atleta-deportista amateur logre controlar sus niveles de estrés, ansiedad, angustia o cualquier otro limitante psicológico que no le permita alcanzar un óptimo desempeño. Un buen manejo de la tolerancia, de la frustración y de la auto-confianza, le servirá en el ámbito deportivo y, además, le permitirá enfrentar cualquier situación problemática o de riesgo que se presente cotidianamente.

La información que entrega el CPRD demuestra que este grupo de atletas-deportistas 
de la ciudad de Concepción, que llevan un tiempo practicando esta modalidad deportiva en distintos grupos y con diferentes entrenadores, desarrollan con éxito su proceso de inserción al deporte, pero si se proyectan hacia la competencia, deberán trabajar la dimensión psicológica, específicamente las características analizadas en el estudio.

\section{REFERENCIAS BIBLIOGRÁFICAS}

Balaguer, I., \& Castillo, I. (1994). Entrenamiento psicológico en el deporte. Valencia: Albatros.

Bandura, A. (1977). Self-efficacy: Toward a Unifying theory of behavioral change. Psychological Review, 2,191-215.

Bandura, A. (1997). La auto-eficacia: El ejercicio del control. Nueva York: Freeman.

Buceta, J. M. (1998). Psicología del entrenamiento deportivo. Madrid: Dykinson.

Cox, R. H. (2009). Psicología del deporte: concepto y sus aplicaciones. Madrid: Editorial Médica Panamericana S.A.

Gimeno, G. \& Buceta, J. M. (2010). Evaluación psicológica en el deporte, el cuestionario CPRD. Madrid: Dykinson.
Gimeno, F., Buceta, J. M., \& Pérez-Llantada, M. C. (2007). Influencia de las variables psicológicas en el deporte de competición: evaluación mediante el cuestionario Características psicológicas relacionadas con el rendimiento deportivo. Psicothema, 19(4), 667-672. Recuperado de https://www.redalyc. org/pdf/727/72719420.pdf

Mahamud, J., Tuero, C., \& Márquez, S. (2005). Características psicológicas relacionadas con el rendimiento: comparación ente los requerimientos de los entrenadores y la percepción de los deportistas. Revista de Psicología del deporte, 14(2), 237-251. Disponible en: https://www. redalyc.org/pdf/2351/235119230004. pdf

Ureña-Bonilla (2015) citado por Gimeno, G. \& Buceta, J. M. (2010). Evaluación psicológica en el deporte, el cuestionario $C P R D$. Madrid, Editorial Dykinson.

Weineck, J. (2000). Salud, ejercicio y deporte activar las fuerzas con un entrenamiento adecuado, prevenir enfermedades con el deporte correcto. Barcelona, Paidotribo.

\section{Dirección para correspondencia}

Prof. Dr. Cristián Luarte Rocha

Director de Pedagogía en Educación Física

Facultad de Ciencias de la Educación

Universidad San Sebastián

Concepción, Chile

Contacto:

cristian.luarte@uss.cl

Recibido: 26-10-2018

Aceptado: 04-12-2018 